\title{
Filtration effects of graphene nanoplatelets in resin infusion processes: \\ Problems and possible solutions
}

Han Zhang ${ }^{\mathrm{a}, \mathrm{b}, *}$, Yi Liu ${ }^{\mathrm{a}}$, Shanshan Huo ${ }^{\mathrm{c}}$, Joe Briscoe ${ }^{\mathrm{a}}$, Wei Tu${ }^{\mathrm{a}, \mathrm{b}}$, Olivier T. Picot ${ }^{\mathrm{a}}$, Amir Rezai $^{\mathrm{d}}$, Emiliano Bilotti ${ }^{\mathrm{a}, \mathrm{b}}$, Ton Peijs $\mathrm{s}^{\mathrm{a}, \mathrm{b}, *}$

a School of Engineering and Materials Science, and Materials Research Institute, Queen Mary University of London, Mile End Road, E1 4NS London, UK

${ }^{\mathrm{b}}$ Nanoforce Technology Ltd., Joseph Priestley Building, Queen Mary University of London, Mile End Road, E1 4NS London, UK

${ }^{c}$ NetComposites Ltd., Unit 4a, Broom Business Park, Bridge Way, S41 9QG Chesterfield, UK

${ }^{\mathrm{d}}$ BAE Systems MA\&I, 20R Buckingham House, Filton, BS34 7QW, UK

Abstract:

Since the discovery of graphene, various industries such as aerospace and automotive are trying to utilize this fascinating nanofiller to enhance components' performance. An important issue in the processing of nanoengineered composites is the interaction and potential filtration of nanofillers by the porous microfibre preform during liquid moulding processing. Here we demonstrate the filtration effect of graphene nanoplatelets (GNPs) during resin infusion of nanoengineered hierarchical composites, and for the first time we have successfully quantified this filtration effect by both electrical and optical methods. In addition, an alternative spraying method to deliver GNPs into composite laminates was also evaluated.

Key words: Graphene nanoplatelets (GNPs); Nanofillers; Epoxy; Liquid Moulding; Filtration; Spray coating.

* Corresponding author. Tel.: +44 02078828865 (T.Peijs); +44 02078822726 (H.Zhang)

E-mail address: t.peijs@qmul.ac.uk; han.zhang@qmul.ac.uk 


\section{Introduction}

Over last few years, fibre-reinforced plastics (FRPs) have been extensively employed in various industries such as aerospace and automotive, replacing traditional metallic materials due to their high specific strength and stiffness. To further explore and utilize FRPs, especially with the aim of improving their relatively weak matrix and interface dominated properties, various nanofillers have been used to create multi-scale nanoengineered composites, including carbon black [1-4], carbon nanotubes (CNTs) [5-9], and graphene nanofillers [10-12].

Due to their extraordinary electrical, mechanical, and thermal properties, graphene and graphene nanoplatelets (GNPs) are among the most attractive nanofillers to be used for such hierarchical composites with enhanced and multi-functional properties, and as a result many research works have been carried out on this topic [13-15]. Interestingly, compared to various successful graphene applications in areas such as batteries, displays, and supercapacitors, the area of FRPs has been rather slow in picking up industrial applications, regardless of the numerous laboratory successes that have been achieved.

One of the main reasons why industrial applications are lagging behind successful academic studies is related to composite processing and potential filtration effects of nanofillers during state of the art liquid moulding processes like resin transfer moulding (RTM) or vacuum assisted resin infusion (VARI). It is generally accepted that a uniform dispersion of nanofillers such as GNPs within epoxy matrices and ultimately composite laminates is essential for enhancement of properties. Moreover, with GNPs as well as other nanofillers like CNTs, even after optimized dispersion in the liquid resin, re-agglomeration during the manufacturing process (i.e. degassing, infusion, curing etc.) may still occur [16]. More importantly, fibre preforms and textiles can act as micro-sized mesh membranes, resulting in 
filtration and filler accumulation in regions close to the resin inlet with less fillers present near the outlets. More obvious filtration effects are expected for CNTs and in particularly entangled networks from CNTs, in comparison to GNPs, and previous research has identified that CNT filtration can occur even at relatively low loadings [17]. This filtration phenomenon, although well recognised, is often ignored in most research works due to the use of small laboratory sized laminates. Unfortunately, in the case of real industrial components part dimensions will be much larger than lab-scale laminates, not to mention the use of highly viscous aerospace grade epoxy resins, where the viscosity will be further increased with the addition of these high aspect ratio nanofillers.

In this study we aim to obtain a better understanding of GNP filtration during liquid moulding of FRPs. For the first time, we have successfully quantified the filtration effect by optical and electrical methods, which have also been confirmed by morphological examinations. Furthermore, a novel and innovative spraying method was evaluated as an alternative solution to deposit and localize GNPs with large lateral dimensions into FRPs without filtration effects.

\section{Experimental}

\subsection{Materials}

The composite system employed in this work consists of a $2 \times 2$ twill E-glass fibre woven fabric (Sigmatex Ltd., UK) and an aerospace grade two component epoxy resin system HexFlow ${ }^{\odot}$ RTM6-2 (Hexcel). Graphene nanoplatelets with a D50 value (medium value of the particle size distribution measured by laser diffraction according to the supplier) of $38 \mu \mathrm{m}$ were supplied by Imerys Graphite \& Carbon (Switzerland) (TIMREX ${ }^{\circledR}$ C-THERM $^{\text {TM}}$ 002).

\subsection{Specimen preparation}


To obtain a homogeneous dispersion of GNPs within the epoxy resin, three roll milling (TRM) was used in this work at a constant speed of $200 \mathrm{rpm}$ and progressively reduced gap distance $(90 / 30,60 / 20,45 / 15,30 / 10$, and $15 / 5 \mu \mathrm{m}$ for back and front gap, respectively), followed by two further dispersion cycles with the parameter of $5 \mu \mathrm{m}$ back gap distance and $15 \mathrm{~N}$ force mode for front gap [18]. Measured amounts of GNPs were added into epoxy resin and dispersed by TRM, while the required amount of hardener was added after TRM processing and prior to the degassing stage.

For the spray coating process, measured amounts of GNPs were dispersed in acetone by an ultrasonication probe (Sonics \& Materials, INC. Model CV334) at $25 \%$ of maximum amplitude and 3000 joules of energy within an ice environment. The spraying setup consisted of an airbrush (Iwata HP-BCS) and air compressor (Iwata studio series) with a 30 psi air pressure and a $10 \mathrm{~cm}$ spraying distance from nozzle to glass fibre fabrics. A more detailed description of the spraying setup can be found in [19].

For the vacuum assisted resin infusion (VARI) process a steel plate treated with mould release agent (Frekote 700-NC) was used as a tool with tacky tape on top to create the mould. With the aim of visualising and quantifying the GNP filtration effect, thin panels consisting of only two plies of fabric were produced. The fabrics were placed next to the resin inlet tubes within the mould, while no flow media was used to eliminate the effect of guided resin flow. The flow distance was marked on the outside of vacuum bag along the middle of the panel with a digital camera recording the resin flow front position as a function of infusion time. A standard curing cycle consisted of heating at a rate of $3{ }^{\circ} \mathrm{C} / \mathrm{min}$ from room temperature to $140{ }^{\circ} \mathrm{C}$ for $1.5 \mathrm{hrs}$, followed by a post cure at $180{ }^{\circ} \mathrm{C}$ for another $2 \mathrm{hrs}$. The thickness of the produced laminates was around $1.4 \mathrm{~mm}$ with a fibre volume fraction between 0.5 and 0.55 . 


\subsection{Characterizations}

\section{Spectrometer}

The optical measurement set-up involved a solar simulator with an output of $2680 \mathrm{Wm}^{-2}$ (Newport model 91160-1000) and a USB2000 fibre optic spectrometer (Ocean Optics). The testing panel was placed in between the solar simulator and spectrometer at a fixed distance. Using this controlled light source at a constant exposure time, the amount of light transmitted through the panel at different locations could be determined by the optic spectrometer at the other end of the panel. Only the panel was moved horizontally during the test to ensure consistent alignment of light source and spectrometer.

\section{Electrical conductivity}

The in-plane electrical conductivity of 1 wt.\% GNP infused laminates was measured by twopoint measurements using a voltage power source (Agilent 6614C) and a picometer (Keithley 6485). Silver paint was applied at both ends of specimens in order to minimise contact resistance.

\section{Morphology}

Scanning electron microscopy (SEM) (Inspect F model, FEI) was used for morphological investigations into the filler distribution in the laminates. Polishing was applied to crosssectional areas of specimens prior to SEM inspections in order to reveal detailed morphologies. Transmission electron microscopy (TEM) (JEOL 2010) was used for further morphological investigations into the actual filler morphologies. For TEM examination the GNP/epoxy mixture after three-roll milling was diluted in acetone and filtered on a nylon membrane $(0.2 \mu \mathrm{m})$ to extract the GNPs. Image $\mathbf{J}$ software was used to measure the size of the interply resin rich pockets as well as the space between fibres within a bundle. 


\section{Rheology}

The rheological properties of reference and GNP filled resins were measured using a rheometer (AR2000) from TA Instrument. Frequency sweeps from $100 \mathrm{rad} / \mathrm{s}$ to $0.1 \mathrm{rad} / \mathrm{s}$ were performed at an isothermal temperature of $90{ }^{\circ} \mathrm{C}$, being the infusion temperature during composite manufacturing. Viscosity values of reference and GNP loaded resins at the same frequency (i.e. $1 \mathrm{rad} / \mathrm{s})$ were taken.

\section{Results and discussions}

\subsection{Morphology}

As-received graphene nanoplatelets (GNPs) were examined under SEM for their lateral dimensions, while GNP fillers after the TRM dispersion process were extracted from the epoxy resin and examined under TEM for thickness measurements, as shown in Fig. 1. As can be seen from the statistical results, most of the fillers have lateral dimensions of around $20 \mu \mathrm{m}$. Fig. 1d shows the edge of GNP flakes under TEM, confirming the thickness of these fillers at around or below $5 \mathrm{~nm}$. These observed values indicate a relatively high aspect ratio of the GNPs of approximately $10^{3}$. Although graphene tends to roll up and wrinkle due to its intrinsic flexibility, it was found that most of the GNP fillers remain flat with a slightly folded edge after three-roll milling. 

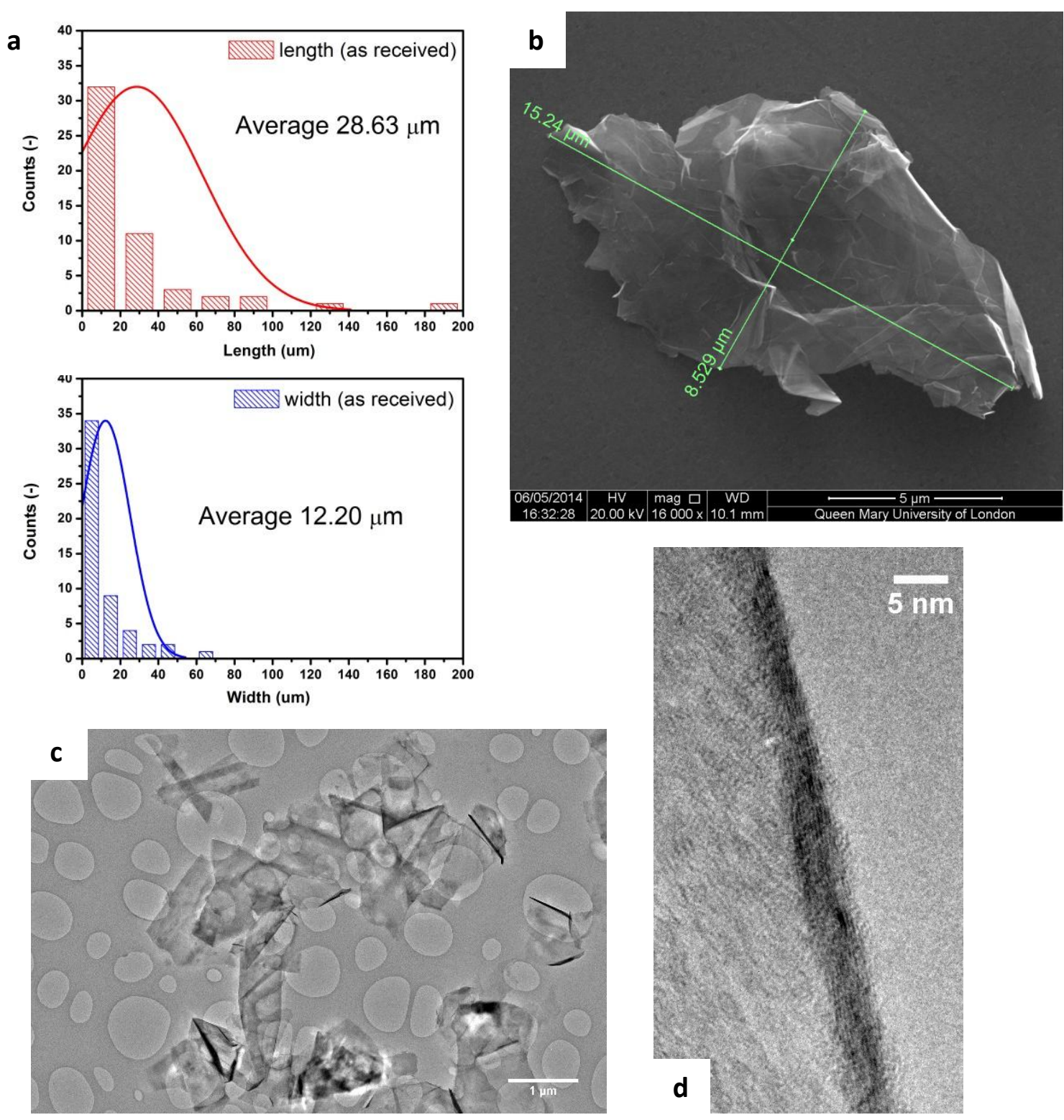

Fig. 1 Statistical lateral dimensions of as-received graphene nanoplatelets (a); SEM images of GNP filler; and TEM images of GNP showing a flake thickness below $5 \mathrm{~nm}$ (c-d).

To avoid an obvious viscosity increase as well as filtration effects relatively low GNP loadings (1 wt.\%) were employed for morphological and electrical studies. Fig. 2 reveals morphological features at cross-sectional areas of the composite laminates. For the case of the reference laminate, clear resin-rich regions were found due to crimp effects in the woven fabrics (Fig. 2a and 2b). These resin pockets with an average size of $0.3( \pm 0.04) \mathrm{mm}^{2}$ are 
potential areas for GNP accumulation, as well as potential locations for GNP network formation. The measured spacing between fibres within a bundle ranged from infinitely small when glass fibres are in contact to up to resin rich regions as large as $100 \mu \mathrm{m}^{2}$.

Two representative sections have been used for morphology comparison between specimens. These include specimens near the resin inlets (Fig. 2c and 2d) and specimens near the resin outlets (Fig. 2e and 2f). Within the resin inlet region, obvious percolated GNP networks can be observed, especially within resin-rich regions (Fig. 2c). Even in areas in between fabric plies where less resin is present, evidence of a GNP network between individual reinforcing fibres was found (Fig. 2d). This is believed to be due to the presence of the reinforcing fabrics which act as a micro-sized mesh for the epoxy/GNP mixture, resulting in more filler to form percolated GNP networks at interply regions as well as intertow regions within fabrics.

On the other hand, for the specimens located near the resin outlets, more fragmented GNPs were observed in resin rich regions with less obvious GNP network formation. Very few traces of GNPs were found within fabric plies with no connected networks present. Apparently, fewer GNPs of relatively small lateral dimensions were able to pass through the micro-sized fabrics, hinting at the existence of a nanofiller filtration effect in the liquid moulding process.

During the resin infusion process, nanofiller within the resin will follow three main flow pathways; (i) interply regions between fabric plies, (ii) intertow spacing within fabric plies, and (iii) intratow spacing between fibres [20, 21]. Clearly, interply regions are the easiest pathway in the case of woven fabrics, especially for fillers with large lateral dimensions. Once these fillers have formed a network and agglomerate within interply regions, other smaller fillers would pass through intertow and eventually intratow spaces. With increasing 
distance away from the resin inlet, the less likely large fillers will go through these spaces.

This is confirmed by morphological observations (see Fig. 2).
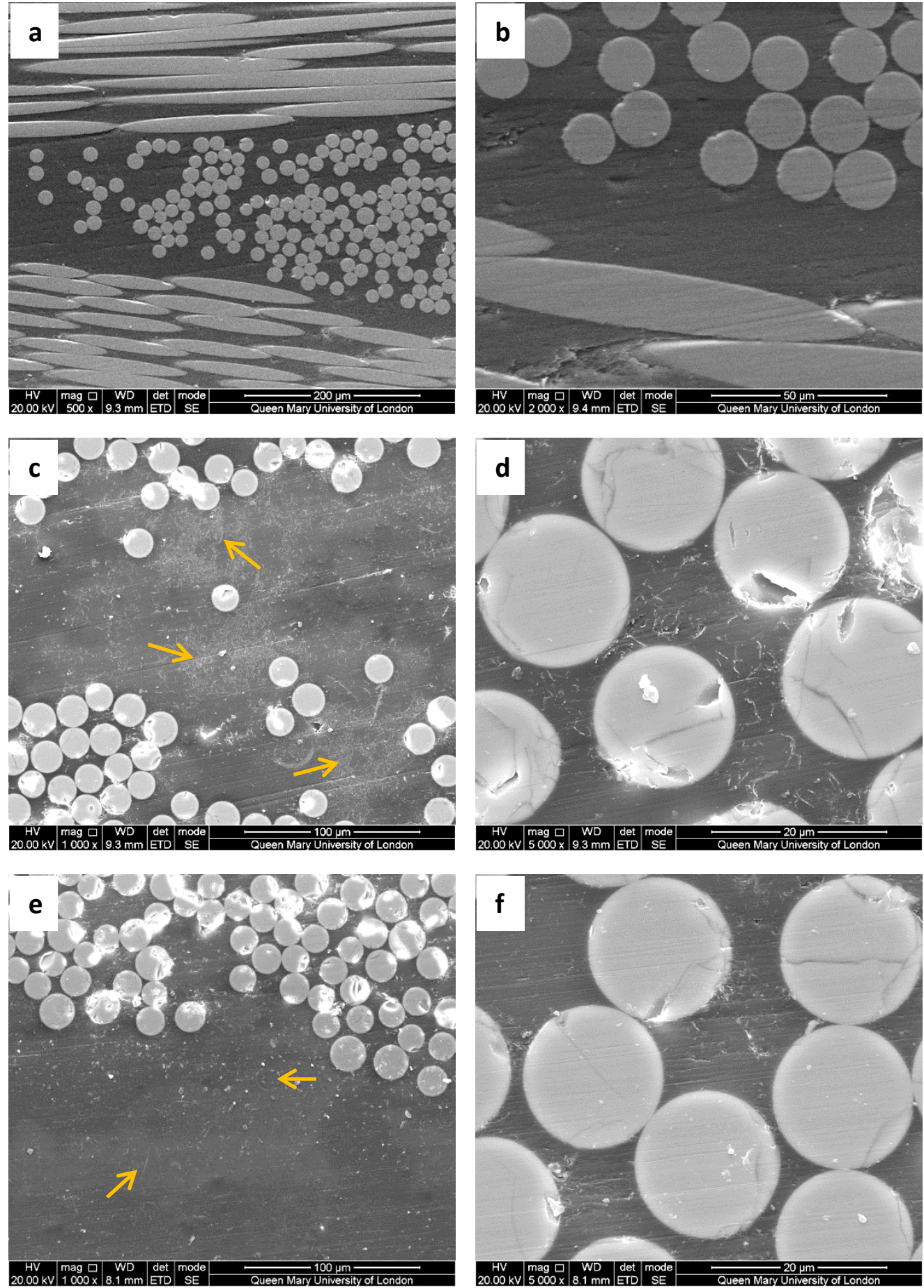

Fig. 2 SEM images of cross-sectional areas for reference and 1 wt. $\%$ GNP modified composites, showing clear resin-rich regions for reference (a)-(b), obvious percolated GNP 
networks for specimens close to the resin inlets (c)-(d), and less well connected GNP networks for specimens close to the resin outlets (e)-(f). Arrows indicate the percolated GNP networks in (c) and trace of GNPs in (e).

\subsection{Optical characterization}

In order to enable the quantification of GNP filtration in composite laminates via optical characterization methods, a certain level of transparency is essential. Unfortunately, the previous panels with $1 \mathrm{wt} . \%$ GNP loading were too opaque to be examined optically. Hence, a lower GNP concentration of $0.5 \mathrm{wt} . \%$ GNP was used for the optical characterizations.

In the optical set-up, the composite laminate is exposed to a light source with a constant output, while the transmitted amount of light is measured by an optical spectrometer on the back-side of the panel. In the case of a good dispersion and distribution of the GNPs within the composite panels (i.e. no filtration), the amount of light transmitted from different locations of the panel should be the same, while areas with higher GNP loadings should possess less optical transparency, hence less transmitted light. Therefore, by analysing the amount of light transmitted at various locations, the relative density of the GNP network and loading can be determined.

Fig. 3a shows the optical transmission spectra at various locations alongside the centre line of the panel, where the spectra from bottom to top were measured from resin inlet towards resin outlet. Apparently the lowest transmitted amount of light was observed at the resin inlet side, while light transmitted slightly increased with increasing distance away from the inlet, until reaching the highest level near the resin outlet. This trend of increasing optical transparency was believed to be due to the presence of fewer GNPs with smaller dimensions away from the resin inlet, which is in agreement with earlier morphological studies (see Fig. 2). 
The highest amount of transmitted light was obtained at a wavelength of $580.67 \mathrm{~nm}$ (Fig. 3a), hence this wavelength was chosen to create a light transmission map for the composite laminates. It is also worth noting that in order to have normalized optical data and to eliminate the effect of the laminate's original transparency values, the transparency at the resin inlet centre position (mid-point of width at resin inlet) was chosen as the reference value. Therefore the transmission map shown in Fig. $3 \mathrm{~b}$ represents the relative change of transmitted light from inlet towards outlet. Fig. $3 b$ shows the optical transmission map at various locations covering the complete area of the composite panel. No obvious differences were observed within a distance up to $120 \mathrm{~mm}$ from the resin inlet, indicating no obvious filtration effects for laminates of this size. However, once the distance away from resin inlet exceeds $120 \mathrm{~mm}$, clear inhomogeneities can be seen in the transmission map, with an overall trend of an increasing amount of transmitted light towards the resin outlet region. These findings are again in good agreement with both morphological observations, as well as the spectra results (see Fig.2 and 3a). 

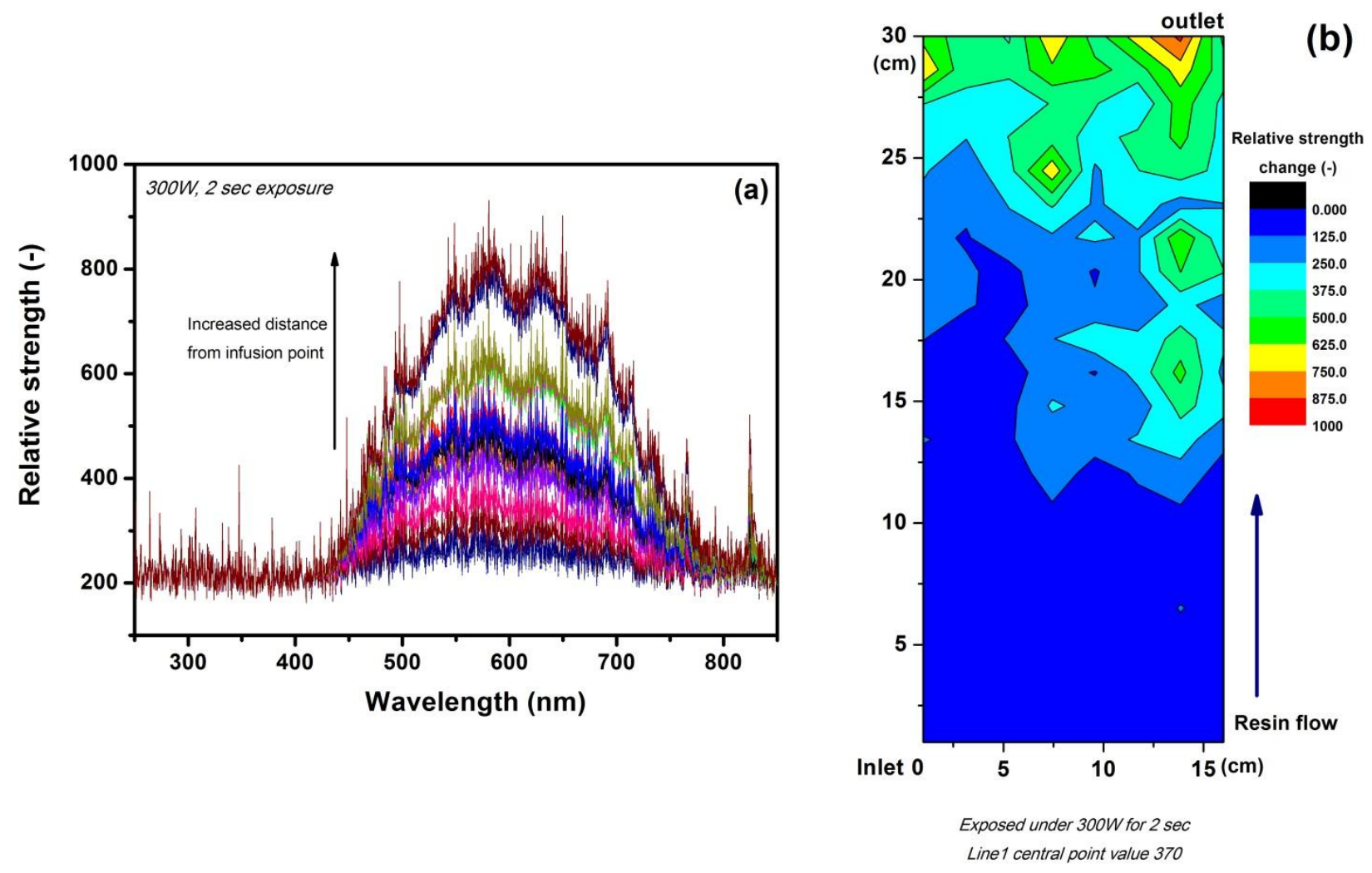

Fig. 3 0.5 wt.\% GNP/RTM6-2 directly infused panel: (a) Centre line optical transmission along the direction of resin flow; (b) Map of transmitted amount of light at various locations (30 cm light source distance for $2 \mathrm{sec}$ at a wavelength of $580.7 \mathrm{~nm}$ ).

Apparently, when the dimensions of composite laminates are below certain values (e.g. 120 $\mathrm{mm}$ for this system), the overall distribution of nanofillers is generally homogeneous without any obvious filtration effects. However, at distances further away from the resin inlet, an uneven filler distribution will be found due to increasing possibilities of GNPs being filtered out by the fibre preform, resulting in lower filler loadings near the resin outlet.

\subsection{Electrical characterization}


The in-plane electrical conductivity was measured for both the $0.5 \mathrm{wt} . \%$ and the $1 \mathrm{wt} \%$ GNP infused panels, with samples cut at different distances from the resin inlet point. It is worth noting that good levels of electrical conductivity were achieved using GNP loadings as low as $1 \mathrm{wt} . \%$, which is attributed to the high aspect ratio of these fillers as well as good levels of dispersion. Even at a relatively low GNP loading of $0.5 \mathrm{wt} \%$, the electrical conductivity reached a level of $10-6 \mathrm{~S} / \mathrm{m}$ for the panel section close to resin inlet. This electrical conductivity was maintained at this level until a distance of around $120 \mathrm{~mm}$ away from the resin inlet, while an obvious reduced electrical conductivity was observed untill a distance of around $200 \mathrm{~mm}$ away from inlet, followed by no measurable electrical properties at further increasing resin flow distance. This trend is believed to be due to filtration effects by the glass fibre preform during resin impreganation, with a large amount of GNPs accumulating in the panel section close to resin inlet, resulting in a higher local GNP concentration and higher electrical conductivity.

Fig.4a shows a good correlation between the electrical conductivity along the panel length and the optical transparency data (see Fig.3b), confirming the filtration effect and effectiveness of the current optical method. With higher GNP loading, as shown in Fig. 4b, electrical conductivity maintained a high level away from the resin inlet untill reaching a distance of around $250 \mathrm{~mm}$ above which an obvious reduction in conductivity was observed with further increasing flow distance. Although the overall electrical conductivity level was not massively altered since local GNP concentrations were all above the percolation threshold, the reducing trend here is in good agreement with the previously reported morphological and optical results (see Fig 2 and 3), confirming a filtration effect during liquid moulding. 

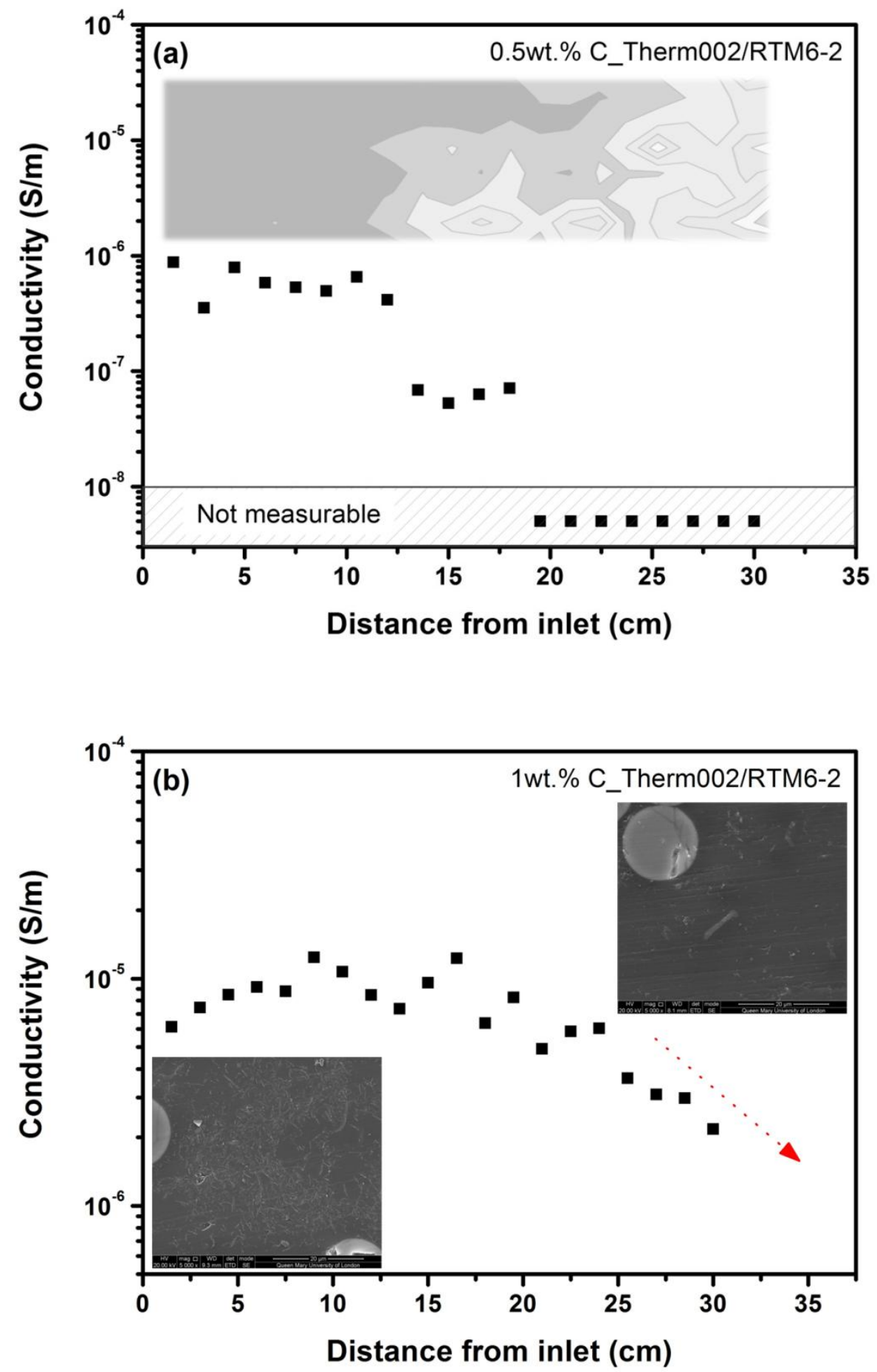

Fig. 4 In-plane electrical conductivity of (a) 0.5 wt.\% GNP infused laminates, with in-set of optical transparency showing a good correlation between electrical and optical results; and (b) 1 wt.\% GNP infused laminates, showing a clear trend of reducing condcutvitity with increasing flow distance away from the resin inlet. 


\subsection{Evaluation of an alternative GNP deposition method}

As mentioned earlier, an alternative spraying method to deposit GNPs into FRPs with the aim of avoiding filtration effects as well as dimensional limitations was also evaluated. There are a number of successful methodologies to introduce nanoparticles into FRPs which avoid filtration issues, including in-situ growth of nanoparticles (i.e. CNTs or graphene) onto reinforcing fibres or fabics by chemical vapour deposition [22, 23], electrophoresic deposition to coat conductive fibre fabrics $[24,25]$, or the direct deposition of nanoparticles

onto fabrics by spray coating techniques [19]. Here we evaluate filler distribution using the simple method of direct spray coating GNPs onto fabrics and subsequently infusing these nano-enhanced fabrics with neat resin to produce a nanoengineered composite laminate. For a fair comparison, the GNP loading for spray coating was calculated to be exactly the same as in directly infused $0.5 \mathrm{wt} . \%$ GNP glass-fibre reinforced laminates.

Fig. 5 shows the optical spectra and transmission map for a spray coated panel with the same amount of GNPs introduced. Compared to the directly infused panel, a much more consistent spectra with little variation from resin inlet to outlet was observed (Fig. 5a), indicating less variability in GNP concentrations along the centre line of the panel. Fig. 5b shows the optical map at different locations of the panel using the same optical strength scale to the directly infused panel (see Fig. 3b). Clearly a much more homogeneous distribution of GNPs was found thoughout this panel. Importantly, no evidence of any "wash out" of GNPs away from the inlet towards the outlet by the resin flow was observed. Clearly, the localization of spray coated GNPs in the interply region was not affected by resin flow during infusion. This is clearly in contrast to the "filtering out" effects observed in directly infused panels, where local filler concentration varies greatly after infusion. It is also worth noting that the optical 
transparency level of spray coated panels was higher than that of direct infused panels, although the total amount of GNPs was the same for both types of panels. Possible reasons for these differences in optical transparency are related to: (i) the difference in GNP orientation within the laminates; and (ii) the location of the GNPs deposited. In the case of direct infusion of GNP filled epoxy, a network of mostly randomly GNPs is formed throughout the laminate with numerous interfaces to diffract the light, leading to a relatively high level of light scattering in through thickness direction. For spray coated samples, most of the GNP fillers are highly aligned in-plane at an interply region, resulting in clear resin rich regions at top and bottom, and less light scattering due to the aligned GNPs.
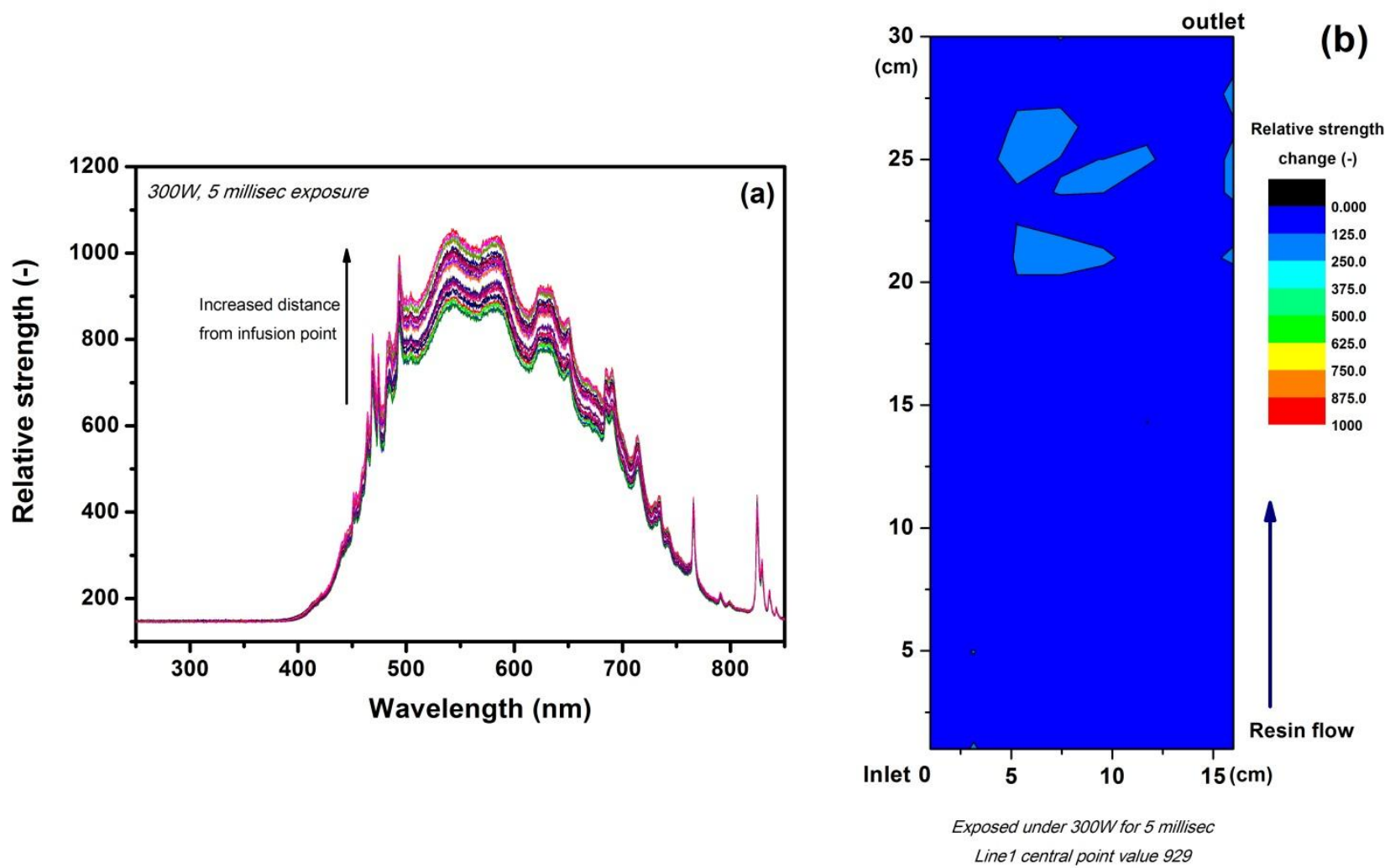

Fig. 50.5 wt.\% GNP/RTM6-2 spray coated panel: (a) Centre line optical transmission along the resin flow direction; (b) Mapping of transmitted amount of light at various locations (30 $\mathrm{cm}$ light source distance for $5 \mathrm{msec}$, at a wavelength of $580.7 \mathrm{~nm}$ ). 


\subsection{Resin front position for permeability study}

Darcy's law (Equation 1) has been widely used to describe resin flow through a porous fibrous medium, where $Q$ is the total discharge, $\kappa$ is the permeability, $\mu$ is the viscosity, and $L$ is the length where pressure drops from values of $P_{b}$ to $P_{a}$. Two main factors need to be considered for filtration effects which are viscosity and permeability. Considering that the viscosity values of neat resin $(0.155 \pm 0.04$ Pa.s $)$ and 0.5 wt.\% GNP modified resin $(0.158 \pm 0.04$ Pa.s $)$ are nearly identical at the infusion temperature of $90{ }^{\circ} \mathrm{C}$, the main difference between the reference and GNP filled panels is permeability. To understand the effect of GNP filtration on permeability during the infusion process, the position of the resin flow front as a function of time has been plotted in Fig. 6a. The permeability of the fibre preforms is determined by interply spaces, intertow spaces, and how these spaces are interconnected, while several modelling and prediction studies have been performed $[20,21$, 26]. Assuming similar resin viscosities and no filtration effects, the resin flow front position between reference and GNP filled panels should be identical. From Fig. 6a, it can be seen that at the beginning of the infusion process, no obvious differences were observed between reference and GNP filled specimens. However, further away from the resin inlet (i.e. above $150 \mathrm{~mm}$ ), an obvious increase in infusion time was observed for GNP filled resins to reach the same position, indicating reduced permeability of the porous fibrous preform structure due to the filtering of GNPs and the formation of agglomerates and networks at the beginning of the mould blocking the resin flow. This was confirmed by analysing the spray coated panel (Fig. 6a) where initial peameability was reduced due to existence of the spray coated GNP network within the glass fibre fabrics while resin viscosity was unchanged as only neat resin was infused, leading to longer infusion times throughout the whole infusion process. 


$$
Q=\frac{\kappa A}{\eta} \frac{\left(P_{b}-P_{a}\right)}{L}
$$
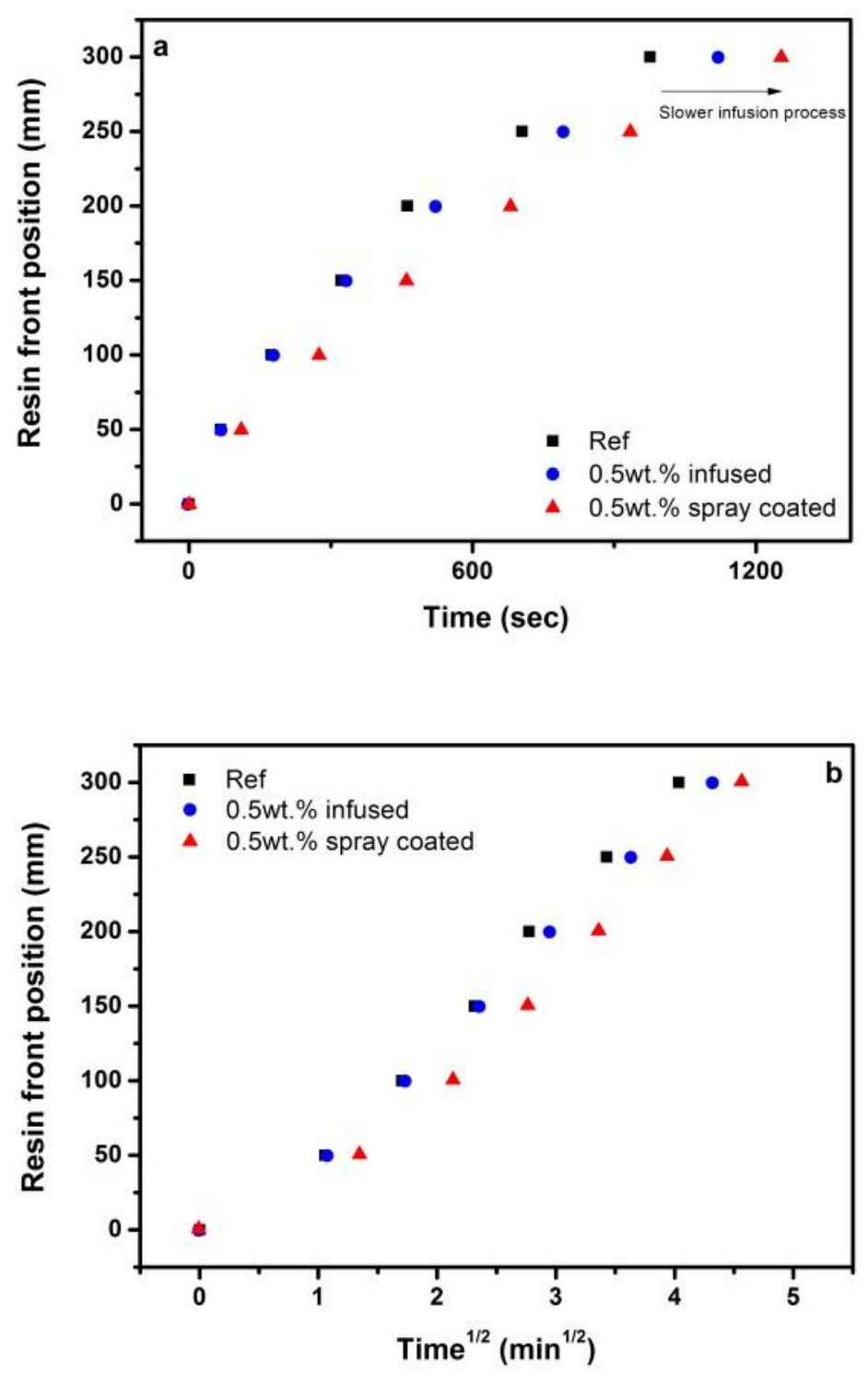

Fig. 6 Resin flow front position during infusion process: (a) as a function of time, and (b) as a function of square root of time, where a linear relationship is expected from Darcy's law.

An important aspect that needs to be considered when analysing the current filtration data is the pressure gradient and non-uniform laminate thickness in the vacuum assisted resin infusion (VARI) process, as the vacuum bag is deformable. This pressure gradient is the main 
cause of the non-uniform thickness, but also the driving force for the resin impregnation in VARI, resulting in non-uniform compaction ratio, permeability, interply and intertow spacings during the filling process. However, even in VARI an equilibrium will occur after resin filling before gelation, leading to a more evenly distributed thickness throughout the final laminate [27-29]. Non-uniform thickness during resin infusion processes remains however an issue and may have affected some of the results presented here. Although controlled thickness processes like resin transfer moulding (RTM) might have solved some of these issues the same factors of varying compaction ratios and pressure gradients have to be addressed there as well [30, 31]. Despite some of its disadvantages, VARI was selected as the manufacturing process for this work as it represents a technology that has gained wide acceptance and increasing usage in both the academia and industry. In fact, the lower level of compaction observed in VARI compared to RTM will lead to higher permeabilities with larger interply spacings, which should lead to more possible pathways for nanofillers and less filtration.

Since both the thickness and width of the mould are much smaller than its length, the resin flow can be largely considered as one-directional in-plane flow. Therefore, a linear dependency of the resin flow front position with the square root of time is expected and used to analyse the resin front position agains time [17]. In Fig. 6b, only a small deviation was observed for the GNP filled panel from the reference panel to the spray coated panel as a result of a reduced permeability, especially for distances exceeding $150 \mathrm{~mm}$ away from the resin inlet. It is worth noting that the distance where the panel based on GNP filled resin started to show filtration effects is consistent with previouly observed results (see Fig, 2, 3 and 4). 


\section{Conclusions}

The filtration effect of GNPs within an aerospace grade epoxy resin in a traditional vacuum assisted resin infusion process has been examined and evaluated. A systematic study including morphological, optical, and electrical property characterizations was performed in order to not only observe and demonstrate the filtration, but also to quantify this effect. It is demonstrated that for the current GNP nanofiller with lateral dimensions of around $20 \mu \mathrm{m}$ in combination with the woven E-glass fibre preform structure and a fibre volume fraction between 0.5 and 0.55 , no obvious filtration was observed up to a flow length of around 120 mm. It is expected that for smaller filler dimensions and/or reduced fibre volume fractions, the flow length (i.e. panel dimensions) above which significant filtration starts to occur may be larger. These observations explains why often no filtration is observed in lab-scale experiments, however it also highlight the significance of the filtration problem in the manufacturing of full scale industrial parts.

To fully utilize these nanofillers for composite applications while avoiding filtration problems during liquid moulding processes, apart from optimized filler dispersion, the following considerations need to be taken into account: (i) the interply distance between the fabric plies, (ii) the intertow distance within the ply, and (iii) the intratow distance between the individual fibres, at a given volume fraction and weave style. This information will determine the regions available for GNP filler localization. As long as the aerodynamic dimension of the filler is smaller than the interply space, it is possible to infuse GNP filled resins to produce good quality composite laminates. It is easy to understand that for smaller filler dimensions, less filtration can be expected. However, typically high aspect ratio nanofillers with large lateral dimensions are preferred for efficient mechanical reinforcement and low percolation thresholds. 
In addtion, an innovative spray coating method for depositing GNPs into composite laminates, while avoiding potential filtration effects was evaluated. Results showed the absence of filtration in a traditional liquid moulding process, when using these spray coated fabrics although it is worth noting that a reduced initial permeability due to the presence of an interply nanofiller network can be expected, leading to slightly increased filling times.

\section{Acknowledgements}

This work was carried out under the framework of the European Research Programme "PolyGraph" (grant agreement number 604143) in the Seventh Framework Programme.

\section{References}

[1] Gojny FH, Wichmann MHG, Fiedler B, Bauhofer W, Schulte K. Influence of nano-modification on the mechanical and electrical properties of conventional fibre-reinforced composites. Compos Pt AAppl Sci Manuf. 2005;36(11):1525-1535.

[2] Zhang D, Ye L, Deng S, Zhang J, Tang Y, Chen Y. CF/EP composite laminates with carbon black and copper chloride for improved electrical conductivity and interlaminar fracture toughness. Compos Sci Technol. 2012;72(3):412-420.

[3] Han S, Chung DDL. Increasing the through-thickness thermal conductivity of carbon fiber polymer-matrix composite by curing pressure increase and filler incorporation. Composites Science and Technology. 2011;71(16):1944-1952.

[4] Nanni F, Ruscito G, Puglia D, Terenzi A, Kenny JM, Gusmano G. Effect of carbon black nanoparticle intrinsic properties on the self-monitoring performance of glass fibre reinforced composite rods. Composite Science and Technology. 2011;71(1):1-8.

[5] Zhang H, Liu Y, Kuwata M, Bilotti E, Peijs T. Improved fracture toughness and integrated damage sensing capability by spray coated CNTs on carbon fibre prepreg. Compos Pt A-Appl Sci Manuf. 2015;70:102-110.

[6] Zhang H, Bilotti E, Peijs T. The use of carbon nanotubes for damage sensing and structural health monitoring in laminated composites: a review. Nanocomposites. 2015;1(4):167-184.

[7] Thostenson ET, Chou T-W. Carbon nanotube networks: Sensing of distributed strain and damage for life prediction and self healing. Adv Mater. 2006;18(21):2837-+.

[8] Zhang H, Liu Y, Bilotti E, Peijs T. In-situ monitoring of interlaminar shear damage in carbon fibre composites. Adv Compos Lett. 2015;24(4):92-97.

[9] Thostenson ET, Ren ZF, Chou TW. Advances in the science and technology of carbon nanotubes and their composites: a review. Compos Sci Technol. 2001;61(13):1899-1912. 
[10] Zhang X, Fan X, Yan C, Li H, Zhu Y, Li X, et al. Interfacial microstructure and properties of carbon fiber composites modified with graphene oxide. ACS Applied Materials and Interfaces. 2012;4(3):1543-1552.

[11] Yavari F, Rafiee MA, Rafiee J, Yu ZZ, Koratkar N. Dramatic Increase in Fatigue Life in Hierarchical Graphene Composites. Acs Applied Materials and Interfaces. 2010;2(10):2738-2743.

[12] Yang X, Wang Z, Xu M, Zhao R, Liu X. Dramatic mechanical and thermal increments of thermoplastic composites by multi-scale synergetic reinforcement: Carbon fiber and graphene nanoplatelet. Materials and Design. 2013;44:74-80.

[13] Zhu Y, Murali S, Cai W, Li X, Suk JW, Potts JR, et al. Graphene and graphene oxide: Synthesis, properties, and applications. Adv Mater. 2010;22(35):3906-3924.

[14] Stankovich S, Dikin DA, Dommett GHB, Kohlhaas KM, Zimney EJ, Stach EA, et al. Graphene-based composite materials. Nature. 2006;442(7100):282-286.

[15] Singh V, Joung D, Zhai L, Das S, Khondaker SI, Seal S. Graphene based materials: Past, present and future. Progress in Materials Science. 2011;56(8):1178-1271.

[16] Inam F, Peijs T. Transmission light microscopy of carbon nanotubes-epoxy nanocomposites involving different dispersion methods. Adv Compos Lett. 2006;15(1):7-13.

[17] da Costa EFR, Skordos AA, Partridge IK, Rezai A. RTM processing and electrical performance of carbon nanotube modified epoxy/fibre composites. Compos Pt A-Appl Sci Manuf. 2012;43(4):593602.

[18] Li Y, Zhang H, Crespo M, Porwal H, Picot O, Santagiuliana G, et al. In Situ Exfoliation of Graphene in Epoxy Resins: A Facile Strategy to Efficient and Large Scale Graphene Nanocomposites. ACS Appl Mater Interfaces. 2016;8(36):24112-24122.

[19] Zhang H, Kuwata M, Bilotti E, Peijs T. Integrated damage sensing in fibre-reinforced composites with extremely low carbon nanotube loadings. J Nanomater. 2015;2015(Article ID 785834):7.

[20] Shih CH, Lee LJ. Effect of fiber architecture on permeability in liquid composite molding. Polym Compos. 1998;19(5):626-639.

[21] Chen ZR, Ye L, Lu M. Permeability Predictions for Woven Fabric Preforms. J Compos Mater. 2010;44(13):1569-1586.

[22] Qian H, Bismarck A, Greenhalgh ES, Shaffer MSP. Carbon nanotube grafted carbon fibres: A study of wetting and fibre fragmentation. Compos Pt A-Appl Sci Manuf. 2010;41(9):1107-1114.

[23] Garcia EJ, Wardle BL, Hart AJ, Yamamoto N. Fabrication and multifunctional properties of a hybrid laminate with aligned carbon nanotubes grown In Situ. Compos Sci Technol. 2008;68(9):20342041.

[24] An Q, Rider AN, Thostenson ET. Hierarchical Composite Structures Prepared by Electrophoretic Deposition of Carbon Nanotubes onto Glass Fibers. ACS Appl Mater Interfaces. 2013;5(6):2022-2032.

[25] Battisti A, Esqué-de los Ojos D, Ghisleni R, Brunner AJ. Single fiber push-out characterization of interfacial properties of hierarchical CNT-carbon fiber composites prepared by electrophoretic deposition. Compos Sci Technol. 2014;95:121-127.

[26] Reia da Costa EF, Skordos AA. Modelling flow and filtration in liquid composite moulding of nanoparticle loaded thermosets. Composites Science and Technology. 2012;72(7):799-805.

[27] Li J, Zhang C, Liang R, Wang B, Walsh S. Modeling and analysis of thickness gradient and variations in vacuum-assisted resin transfer molding process. Polym Compos. 2008;29(5):473-482.

[28] Yu BM, Lee LJ. A simplified in-plane permeability model for textile fabrics. Polym Compos. 2000;21(5):660-685.

[29] Chohra M, Advani SG, Gokce A, Yarlagadda S. Modeling of filtration through multiple layers of dual scale fibrous porous media. Polym Compos. 2006;27(5):570-581.

[30] Lebrun G, Gauvin R, Kendall KN. Experimental investigation of resin temperature and pressure during filling and curing in a flat steel RTM mould. Compos Pt A-Appl Sci Manuf. 1996;27(5):347-356.

[31] Deleglise M, Binetruy C, Krawczak P. Solution to filling time prediction issues for constant pressure driven injection in RTM. Compos Pt A-Appl Sci Manuf. 2005;36(3):339-344. 